\title{
Anatomic and Functional Development of the Suprachiasmatic Nuclei in the Gray Short-Tailed Opossum
}

\author{
Scott A. Rivkees, ${ }^{1}$ Charles A Fox, ${ }^{2}$ Carol D. Jacobson, ${ }^{2}$ and Steven M. Reppert' \\ 'The Laboratory of Developmental Chronobiology, Children's Service, Massachusetts General Hospital and Harvard \\ Medical School, Boston, Massachusetts 02114, and 'Department of Veterinary Anatomy, lowa State University, Ames, \\ lowa 50011
}

\begin{abstract}
The anatomic and functional development of the suprachiasmatic nuclei (SCN) was studied in the gray short-tailed opossum, Monodelphis domestica. To provide a background for developmental studies, daily patterns of wheel-running behavior and SCN metabolic activity were studied in adult animals. Adult opossums manifested robust circadlan rhythms in locomotor activity that were entrained by the daily lightdark cycle. The temporal course of SCN metabolic activity, studied by the ${ }^{14} \mathrm{C}$-labeled deoxyglucose autoradiographic technique, showed that the adult SCN were metabolically active throughout subjective day and relatively inactive during subjective night. SCN neurogenesis, determined using ${ }^{3} \mathrm{H}$-thymidine autoradiography, was active at postnatal day 3, the earliest age studied, and continued until postnatal day 7. It was not until postnatal day 16 that the SCN appeared as distinct nuclei by light microscopy. Study of the ontogeny of the daily rhythm in SCN metabolic activity showed that the nuclei were metabolically active during both day and night at postnatal day 16 . On day 20 , a clear day-night rhythm in SCN metabolic activity was first observed; the rhythm was even more pronounced on day 27 . These results indicate that the gray short-tailed opossum has a functioning circadian timing system and that the anatomic and functional development of the $\mathrm{SCN}$ in this species occurs during the postnatal period.
\end{abstract}

Circadian rhythms are endogenously generated daily rhythms that are driven by a circadian pacemaker or biological clock. The suprachiasmatic nuclei (SCN) of the anterior hypothalamus are the only site known to function as a circadian pacemaker in the mammalian brain (Takahashi and Zatz, 1982; Moore and Card, 1985). To maintain synchrony of internal rhythmic functions with the outside world, the daily light-dark cycle entrains the SCN pacemaker to the $24 \mathrm{hr}$ period. Light information reaches the SCN primarily via the monosynaptic retinohypothalamic tract (Moore, 1973).

\footnotetext{
Received Dec. 8, 1987; revised Feb. 12, 1988; accepted Feb. 15, 1988.

We thank Andrew Dowd, Tom Larsen, and Ruth Larsen for expert technical assistance; Drs. David Weaver and John Nadakavukaren for reviewing the manuscript; and Dr. Gregory Crosby, Anesthesia Research, Massachusetts General Hospital, for use of his image analysis system for assessment of $\mathrm{dG}$ autoradiographs. This work was supported by PHS Grants HD14427 (S.M.R.) and HD16148 (C.D.J.), March of Dimes Basic Research Grant 1-945 (S.M.R.), and the Pediatric Career Physician Scientist Program Grant U10-HD22297 (S.A.R.). S.M.R. is an Established Investigator of the American Heart Association.

Correspondence should be addressed to Dr. Rivkees \% Dr. Reppert at the above address.

Copyright (C) 1988 Society for Neuroscience $0270-6474 / 88 / 114269-08 \$ 02.00 / 0$
}

One technique used for establishing the pacemaker function of the $\mathrm{SCN}$ is the ${ }^{14} \mathrm{C}$-labeled deoxyglucose $(\mathrm{dG})$ autoradiographic method (Schwartz and Gainer, 1977; Schwartz et al., 1980). This method provides an in vivo assessment of the rates of glucose utilization (metabolic activity) of individual brain structures (Sokoloff, 1977). The SCN of several mammals exhibit a clear daily rhythm of glucose utilization, with the nuclei being metabolically active during the day and inactive at night (Schwartz et al., 1983).

The $\mathrm{dG}$ method is especially useful for studying SCN function during early development. Prior to the overt expression of circadian rhythms, a daily rhythm in SCN metabolic activity can be detected using the $\mathrm{dG}$ technique. Previous $\mathrm{dG}$ studies in rats have shown that the developing biological clock in the $\mathrm{SCN}$ is oscillating prcnatally (Rcppert and Schwartz, 1983, 1984). An interesting aspect of this circadian development is that during late fetal and early neonatal life, before the retinohypothalamic tract innervates the SCN, the dam entrains the developing animal to the prevailing lighting conditions (Reppert et al., 1987).

Our current knowledge of the mammalian "circadian timing system" (the system responsible for the generation, regulation, and expression of circadian rhythms) and its development is based largely on studies of eutherian mammals. These animals have an elaborate placenta for supporting extended in utero development. Little information is available, on the other hand, about the circadian timing system of metatherian species (which include marsupials). In these species, the offspring are extremely immature at birth, and early stages of postnatal development are equivalent to the fetal stages of most eutherian animals (Hartman, 1952; Jacobson, 1984; Jacobson et al., 1985). Metatherian animals thus provide an interesting comparative approach for investigating the ontogeny of the circadian timing system. The gray short-tailed opossum is especially well suited for such studies; these animals are small (adults weight 75-150 $\mathrm{gm}$ ) and easily maintained in a laboratory setting, and the species is pouchless, making the pups readily accessible to the investigator (Fadem et al., 1982; Cothran et al., 1985).

This paper describes the anatomic and functional devclopment of the SCN in the gray short-tailed opossum. Because the circadian timing system has not been extensively studied in marsupials, we first characterized circadian rhythms in locomotor behavior and SCN metabolic activity in adult animals. With this background, we next examined the neurogenesis and morphogenesis of the developing SCN. Finally, we determined when the circadian rhythm in SCN metabolic activity is first apparent in developing pups. 


\section{Materials and Methods}

Animals. Adult gray short-tailed opossums (Monodelphis domestica) from the Southwest Foundation for Biomedical Research (San Antonio, TX) were used. Animals were housed individually in plastic cages that were maintained in temperature-controlled animal care facilities $\left(24^{\circ} \mathrm{C}\right)$ Unless otherwise specified, the animals were kept on an automated diurnal light-dark cycle (LD) consisting of $14 \mathrm{hr}$ of light/d. Food (Reproduction Fox Chow; Milk Specialty Products, Madison WI) and water were available at all times. Lactating dams were also given bran cereal (All Bran; Kelloggs, Battle Creek, MI) to help prevent rectal prolapse that can occur during lactation. A plastic container in each female's cage served as a nest box. For breeding, male and female were paired for 9-14 d. Animals were then separated and the dam checked daily for the presence of pups (day of birth = day 0 ). The gestation period is 14 $15 \mathrm{~d}$ (Fadem et al., 1982), and birth generally occurs $10 \mathrm{~d}$ after the termination of pairing.

For Experiments 1, 2, and 5, in which strict control over ambient lighting conditions was necessary, animals were housed individually in clear plastic cages $(25 \times 15 \times 15 \mathrm{~cm})$ within well-ventilated, lightproof compartments. Each compartment had its own timer that controlled the $\mathrm{LD}$ cycle within. During the light portion of the LD cycle, illumination was provided by $20 \mathrm{~W}$ cool white fluorescent tubes (Westinghouse; Pittsburg, PA) delivering an intensity of about 600 lux at the midcage level. For the dark portion of the LD cycle and during constant darkness, red light (wavelength $>620 \mathrm{~nm}$ ) was provided by $20 \mathrm{~W}$ litho no. 2 fluorescent tubes (Chemical Products, North Warren, PA) with an intensity of $0.8 \mu \mathrm{W} / \mathrm{cm}^{2}$. The dim red light helped facilitate animal care and experimental manipulations in "darkness."

Monitoring and analysis of running wheel behavior. Running wheels (22 cm diameter) were mounted on an axle that extended across the width of the cage. With each wheel revolution, a plexiglass triangle on one end of the axle depressed a switch, closing an electrical circuit and producing an electrical event. The events were monitored by an IBM computer-based automated data-collection system (Dataquest III, Minimitter/Data Sciences, Sunriver, OR). Data were graphically expressed by a computer-generated actogram. Behavior rhythms were assessed visually, and the phase of activity onset was determined by drawing an eye-fitted line through activity onset over successive days of an actogram (Pittendrigh and Daan, 1976).

$d G$ Methodology. Animals were maintained in LD for at least 2 weeks prior to study; they were then placed in constant darkness on the day of study. At specified ages and clock times (see below), animals were injected with $125 \mu \mathrm{Ci} / \mathrm{kg}$, i.p., of ${ }^{14} \mathrm{C}$-2-deoxy-D-glucose (Amersham, Arlington Heights, IL; sp act, $60 \mathrm{Ci} / \mathrm{mol}$ ). All dG injections were performed in the dark, and the animals were kept in darkness until killed. Intraperitoneal injections were used for all animals because intracardiac or intravenous injections were not feasible because of the small size of the pups. Injection volumes for newborn and adult animals were 15 and $200 \mu \mathrm{l}$, respectively. Adult animals were manually restrained during injections. For injection of pups, offspring were removed from the nipple by gentle traction while the dam was swaddled. Following injection, adult animals were returned to their cages, while the pups were placed in a small cup positioned on a heating block maintained at $30^{\circ} \mathrm{C}$.

At $45 \mathrm{~min}$ after injection, each animal was killed by decapitation. Brains were removed from the calvaria with the aid of a magnifying visor, frozen in cooled 2-methylbutane $\left(-20\right.$ to $\left.-30^{\circ} \mathrm{C}\right)$, and stored at $-80^{\circ} \mathrm{C}$. Brains were embedded in $\mathrm{M}-1$ embedding matrix (Lipshaw, Detroit, MI), and serial coronal sections $(20 \mu \mathrm{m})$ were cut in a cryostat at $-20^{\circ} \mathrm{C}$, with assessment of anatomical features by dark-field microscopy. Sections were thaw-mounted onto glass slides and dried on a hot plate. Autoradiographs were obtained by exposing the sections to Kodak SB-5 x-ray film for 7-14 d. Sections were stained with cresyl violet. The location of the SCN was histologically identified by light microscopy.

To assess SCN metabolic activity, optical densities (OD) of the SCN were obtained using the Drexel University Image Processing Center "Brain Software Package," run on an IBM AT computer, with a Circon MV 9015-H monochrome microvideo camera. For each brain, the OD of 4 consecutive sections in the midportion of the SCN was determined; the data for each brain werc then avcraged. For the pups, the region of the autoradiograph corresponding to the $\mathrm{SCN}$ was determined by superimposing a computer-generated image of a cresyl violet-stained section over a computer-generated image of the corresponding autoradiograph. For adults, the location of the SCN was assessed by light-microscopic analysis of the cresyl violet-stained sections. The OD of hypothalamic tissue adjacent to the SCN was also measured for each section, allowing expression of the data as relative OD (OD of SCN/OD of adjacent hypothalamus). The use of relative OD is an internal control that corrects for differences in the amount of dG administered, section thickness, and exposure time between animals, thus permitting comparisons among different animals (Sharp et al., 1983).

Neurogenesis methodology. Each litter of pups was injected at a different postnatal age between days 3 and 12 . Individual animals were injected subcutaneously along the dorsal midline with $30 \mu \mathrm{l}$ of ${ }^{3} \mathrm{H}-$ thymidine (Amersham; $52 \mathrm{Ci} / \mathrm{mmol}$ ), while the dam was manually restrained. The injection sites were sealed with collodion. Pups were ancsthetized by hypothermia at days $28-30$ of postnatal life, and perfused by intracardiac injection of $10 \%$ neutral-buffered formalin. The head was removed and placed in Bouin's fixative for $24 \mathrm{hr}$. Daily changes of $10 \%$ buffered formalin were used to clear tissues of Bouin's fixative. Brains were then removed from the calvaria and placed in buffered formalin. Autoradiographs were then generated, as previously described (Jacobson and Gorski, 1981). Brains were embedded in Surgipath tissue medium, and serial coronal sections $(6 \mu \mathrm{m})$ approximating that of the deGroot (1959) plane for the rat were obtained. Slides were dipped in NTB-2 nuclear track emulsion (Kodak) diluted 1:1 with distilled water and kept in lightproof, dessicated boxes at $4^{\circ} \mathrm{C}$. for $30 \mathrm{~d}$. Slides were developed with Kodak Dektrol and fixer and stained with cresyl violet.

For each day of injection, a minimum of 3 animals from each litter was analyzed. All sections that contained the SCN were determined and recorded for each opossum. To sample the entire nucleus, the sections lying along one-quarter, one-half, and three-quarters of the full rostralcaudal extent of the nucleus on the left side of the brain were chosen for analysis. The data presented are the averages obtained from the 3 sections of each brain. The slides were then coded and randomized so that further analyses were performed without knowledge of treatment.

Analyses were performed with dark-field illumination using a Zeiss universal microscope. One investigator viewed each of the sections and determined the approximate percentage of labeled cells within the SCN. To quantify these results, the microscope was interfaced with a DAG MTI 68 video camera attached to a Zeiss SEM IPS image-analysis system. The area occupied by silver grains was determined, as was the total area of SCN cell bodies.

Morphogenesis analysis. To determine when anatomical differentiation of the SCN takes place, serial coronal sections of the hypothalamus were examined by light microscopy. Pups of known age from 0 to 27 d were decapilated and the heads immersion-fixed in neutral-buffered formalin. Following $24 \mathrm{hr}$ of fixation, the calvaria of pups older than $10 \mathrm{~d}$ of age were incised to aid in fixation of the brain. Prior to embedding, the calavaria were decalcified. Brains were embedded in Surgipath tissue medium, serially sectioned ( $6 \mu \mathrm{m}$ coronal), and stained with cresyl violet or hematoxylin and eosin.

\section{Results}

\section{Experiment 1: description of a circadian rhythm in locomotor} activity

Because circadian rhythms have not previously been studied in this species, we first examined the daily pattern of locomotor activity in adults. Locomotor activity was chosen for investigation because of extensive studies in rodents demonstrating that it is a reliable and convenient output of the circadian timing system and can be continuously monitored in individual animals (Moore-Ede et al., 1982). Two 60-d-old male animals (M1, M2) and one female animal (F1) were housed individually in cages that were maintained in a compartment without other animals. To avoid cyclic temporal cues, the compartment was opened in darkness only every 3-4 d, and the time of day at which the compartment was opened was randomized. Wheelrunning activity of each animal was continuously monitored for $20 \mathrm{~d}$ in LD (lights on from 0500 to 1900 hours), for $40 \mathrm{~d}$ in constant darkness (DD), $26 \mathrm{~d}$ in a reversed light-dark cycle (DL; lights on from 1900 to 0900 hours), and $25 \mathrm{~d}$ in DD.

In LD, all animals displayed clear daily rhythms in wheelrunning behavior, with activity occurring predominantly during 

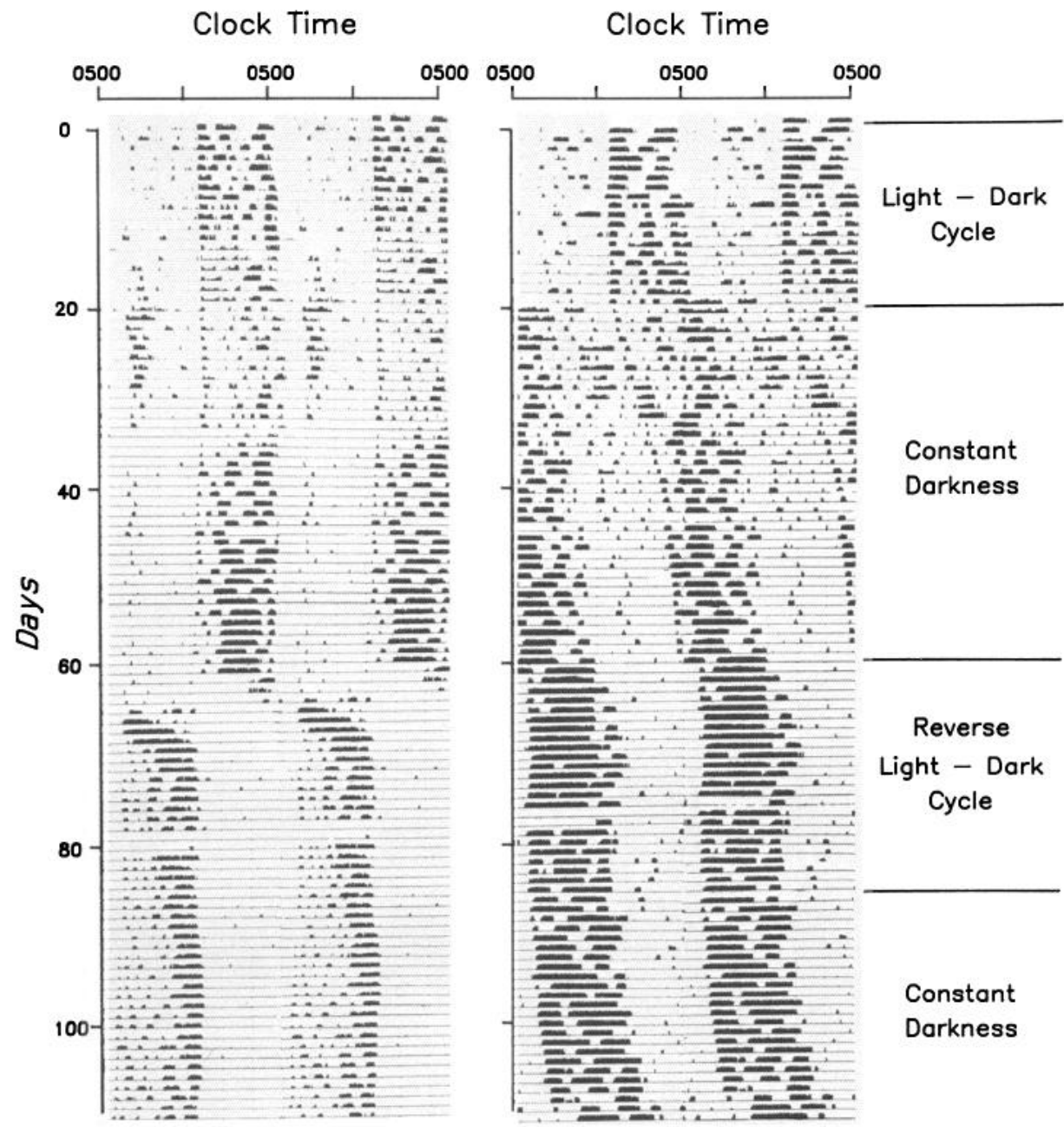

Figure 1. Double plots of locomotor activity in adult opossums maintained in various lighting conditions. Anima F1 (left-hand column) and animal M1 (right-hand column) were housed sequentially in a light-dark cycle (20 d), constant darkness (40 d), a reversed light-dark cycle ( $26 \mathrm{~d})$, and in constant darkness $(25 \mathrm{~d})$. Each horizontal line represents side-by-side plots of consecutive $24 \mathrm{hr}$ periods of activity, with successive days plotted below each other. Vertical deflections represent episodes of wheel running. darkness (Fig. 1). The major bout of activity began with the onset of darkness and promptly ceased when light came on in the morning. Scattered episodes of activity were also noted during the light portion of the LD cycle.

To determine whether the daily activity pattern observed in LD was an endogenously generated circadian rhythm, the lighting cycle was changed to DD. In the constant environment, the animals continued to manifest robust daily activity rhythms (Fig. 1). The duration of the activity period during subjective night (the time in constant darkness when the lights would have been off had the animals remained in a LD cycle) was similar to that seen during the dark portion of LD. Immediately after the lighting was changed to DD, the amount of activity during subjective day (the time in constant darkness when the lights would have been on had the animals remained in a LD cycle) increased compared to that observed in LD. Over successive days in DD, however, there was decreased activity during subjective day. In DD, the cycle length of the locomotor activity rhythm of animal M1 was greater than $24 \mathrm{hr}$, resulting in a progressive, constant drift of phase of activity onset from that observed in LD. After several days in DD, the period length of the activity rhythm decreased from $25.0 \mathrm{hr}$ (days 20-40) to 24.5 $\mathrm{hr}$ (days 40-60). The period length of the activity cycle of animal F1 was $24 \mathrm{hr}$ in DD.

To determine whether the locomotor activity in these animals was entrained by the light-dark cycle, the lighting cycle was acutely changed from DD to DL. In DL, the activity pattern of animal M1 quickly shifted to the new lighting cycle within $1 \mathrm{~d}$ because the animal's circadian phase was already close to DL at the time of the change in lighting (Fig. 1). In animal F1, the activity pattern took 1 week to realign itself to DL, after which time a stable phase relationship was established between the animal's activity pattern and the new lighting cycle. When the animals were placed back into DD, the rhythms persisted from the phases established in DL. The activity patterns of animal M2 were similar to those of animal F1 for all lighting conditions examined (data not shown).

\section{Experiment 2: demonstration of a circadian rhythm in SCN metabolic activity in adult opossums}

We next examined the daily pattern of SCN metabolic activity in adult animals. These studies were performed because SCN metabolic activity has not been previously examined in a marsupial. In addition, the dG method was used exclusively to monitor the circadian timing system during early development in the experiments described below. Four animals were injected with dG during subjective day at 0700,1200 , and $1700 \mathrm{hr} ; 3$ animals were injected during subjective night at 2100,2400 , and $0300 \mathrm{hr}$.

The gross morphology of the SCN was studied by light-microscopic examination of the cresyl violet-stained coronal sections used to generate the autoradiographs. The $\mathrm{SCN}$ in this 
Figure 2. Autoradiographs of coronal brain sections from adult opossums injected with dG during subjective day (top) or subjective night (bottom). The metabolically active SCN appear as a pair of dark spots during subjective day (arrow), while at night images of the $\mathrm{SCN}$ are not visible. Sections are from the region of the mid-SCN. Scale bar, $1 \mathrm{~mm}$.

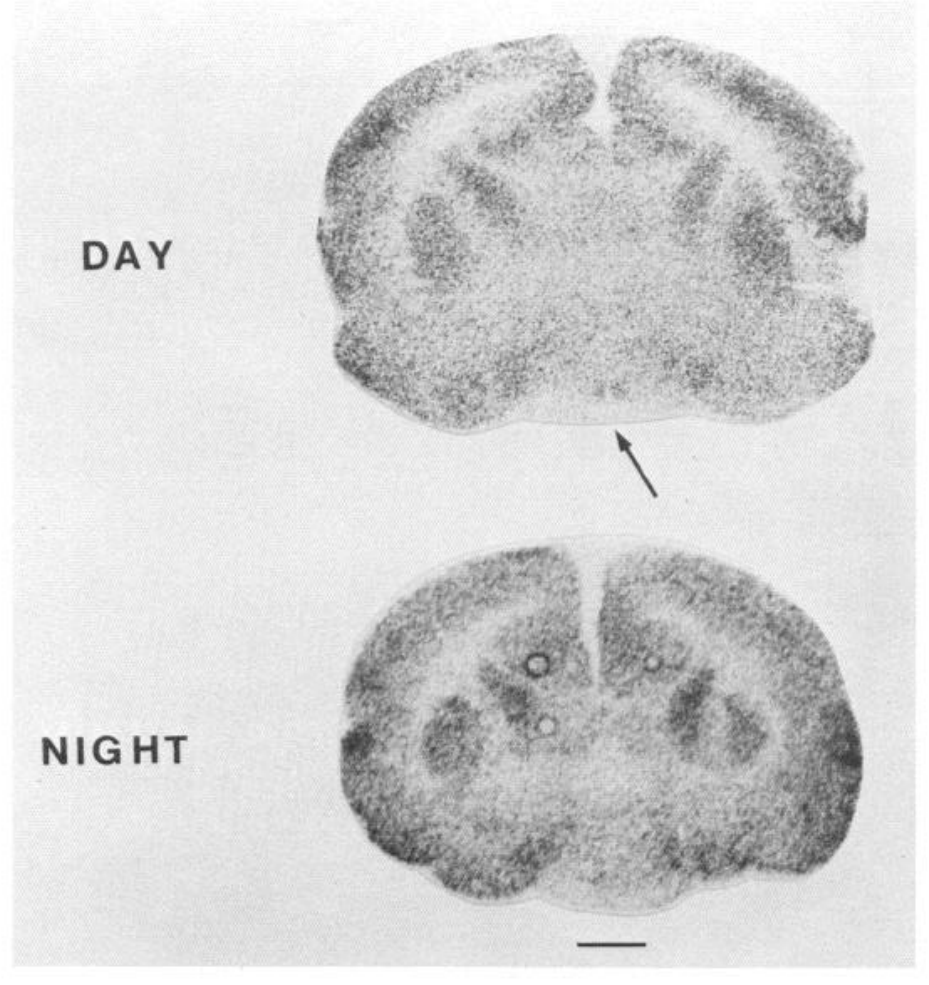

species were easily identified just above the optic chiasm, at the base of the third ventricle. They were first apparent about 150 $\mu \mathrm{m}$ posterior to the start of the optic chiasm, where the chiasm begins to flatten. The SCN ended approximately $150 \mu \mathrm{m}$ past the point where the optic chiasm separates into 2 distinct optic tracts.

Analysis of the autoradiographs showed that during subjective

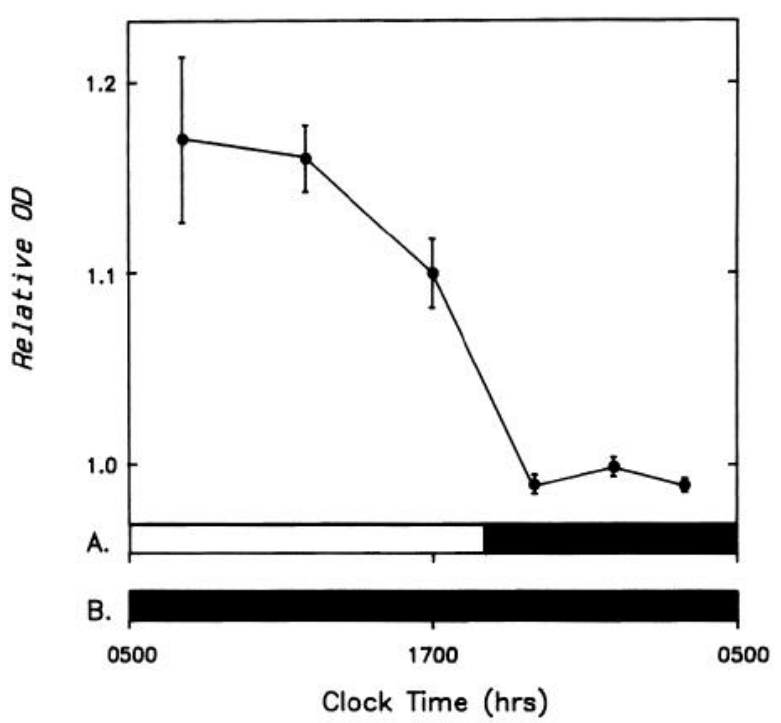

Figure 3. Daily pattern of SCN relative optical densities (OD of SCN/ OD of adjacent hypothalamus) of adult male opossums. The mean relative OD \pm SEM for $3-4$ animals is depicted at each time point (0700, 1200, 1700, 2100, 2400, and 0300 hours). $A$, Light-dark cycle before study. $B$, The animals were studied in darkness. Solid area of horizontal bars represents darkness. day, the autoradiographic images of the SCN were clearly visible as dark ovoid densities throughout the anterior to posterior extent of the nuclei (Fig. 2). During subjective night, the SCN were not visible on the autoradiographs. Relative OD values confirmed the visual assessment of a clear circadian rhythm in $\mathrm{SCN}$ metabolic activity in this species (Fig. 3). The subjective day OD values were similar at 0700 and $1200 \mathrm{hr}$. However, the OD values $2 \mathrm{hr}$ before the onset of subjective night $(1700 \mathrm{hr})$ were smaller than at the preceding time points. The average subjective night $\mathrm{OD}$ values were similar at each of the 3 time points examined.

\section{Experiment 3: neurogenesis of the SCN}

The thymidine autoradiography technique was used to determine the time period in which neurons destined to be $\mathrm{SCN}$ cells are formed. Neurogenesis of the anterior hypothalamus has not previously been studied in this species. Results indicate that neurogenesis of SCN neurons and their precursors was occurring at the earliest day studied (postnatal day 3). Qualitative and quantitative analysis of emulsion autoradiographs indicated that the number of silver grains overlying the $\mathrm{SCN}$ as the result of ${ }^{3} \mathrm{H}$-thymidine uptake progressively decreases until postnatal day 7 (Figs. 4,5 ). This finding was confirmed by directly determining the approximate number of labeled cells in the $\mathrm{SCN}$ using light microscopy and a Zeiss image-analysis system to compute (1) the area of the SCN (cell bodies and neuropil) with overlying silver grains, and (2) the area of cell bodies with overlying silver grains (data not shown). In contrast to the time course of SCN cell formation, neurogenesis of the hypothalamus dorsal to the $\mathrm{SCN}$ continues for a long duration of time, as is evident in Figure 4. This agrees with previous findings (Larsen and Jacobson, 1985). Thus, it appears that the temporal pattern of hypothalamic neurogenesis proceeds from the ventral to the dorsal hypothalamus. 


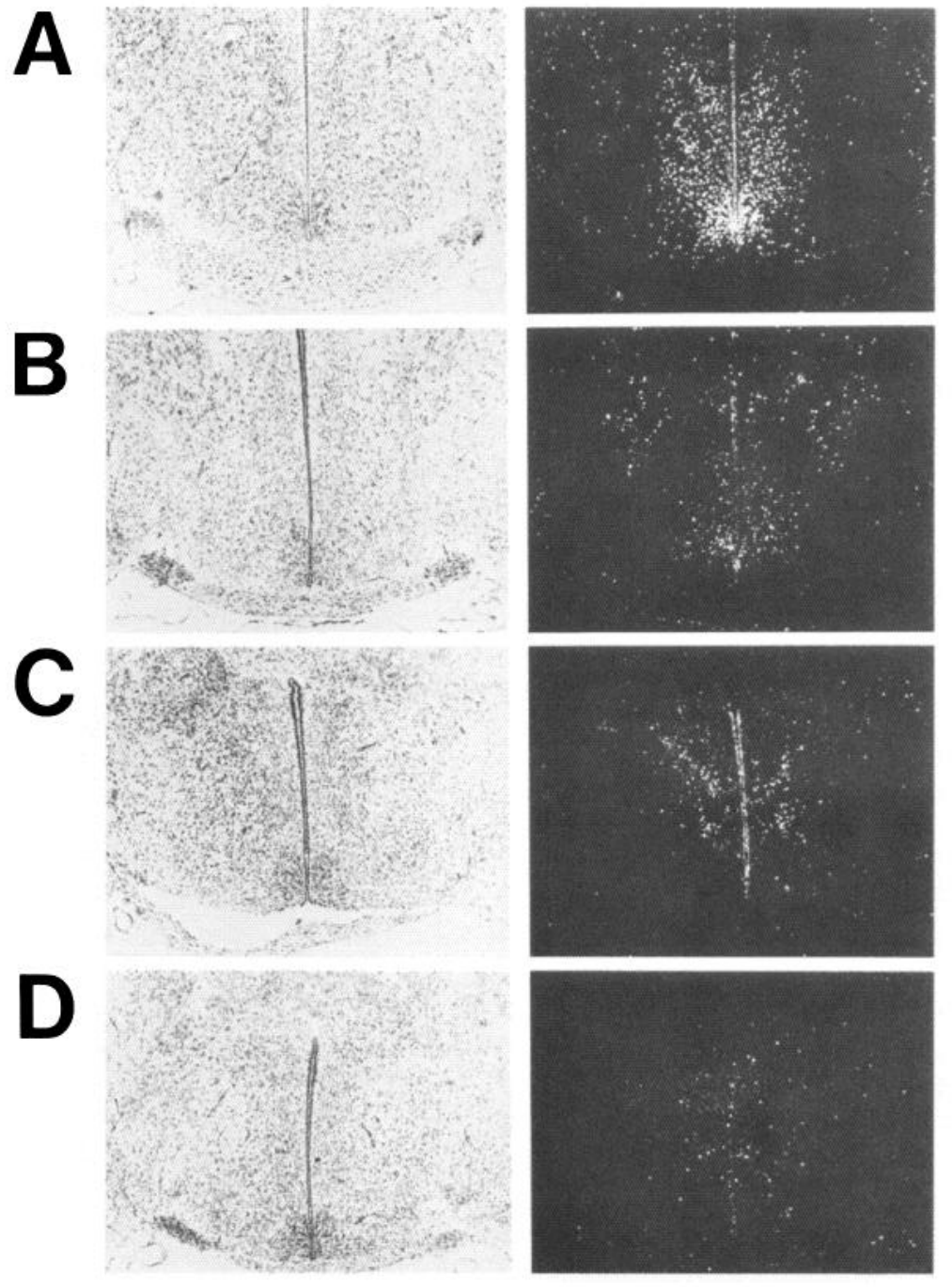

Figure 4. Bright-field (left) and darkfield (right) photomicrographs of 30-dold opossums previously injected with ${ }^{3} \mathrm{H}$-thymidine on postnatal day $4(A), 6$ $(B), 8(C)$, or $10(D)$. Increased grain number (whiteness) corresponds to increased thymidine uptake. Scale bar, 1 $\mathrm{mm}$.

\section{Experiment 4: morphology of the developing SCN}

As a background for functional studies of the developing SCN, we characterized the anatomical differentiation of these nuclei. Cresyl violet-, and hematoxylin- and eosin-stained brain sections were examined by light microscopy to ascertain the age at which the SCN could be readily distinguished from the adjacent hypothalamus. On the day of birth, the brain was not well differentiated and resembled an expanded neural tube without a recognizable hypothalamus. The $\mathrm{SCN}$ were first discernible as distinct nuclei on day 16 of life (Fig. 6, top right-hand column). The dorsal and lateral borders of the SCN did not become distinct until day 27 , when the anterior hypothalamus resembled that of the adult opossum.

\section{Experiment 5: development of a day-night rhythm in SCN metabolic activity}

The dG technique is the only practical method for monitoring the circadian timing system during early development; pups are not weaned until $60 \mathrm{~d}$ of age, which precludes the monitoring of locomotor activity. For this study, one-half of the pups from each litter were injected with dG at mid-subjective day (1300 $\mathrm{hr}$ ); the remaining pups were injected at mid-subjective night $(2300 \mathrm{hr})$. These time points were chosen on the basis of the pattern of SCN metabolic activity in adults delineated in Experiment 2 . SCN metabolic activity was first assessed on day 16 , because this is the earliest age at which the $\mathrm{SCN}$ are apparent as discrete nuclei by light microscopy (see above). Animals were also studied at 20 and $27 \mathrm{~d}$ of age. On day $16,5-7$ pups from each of 2 litters were studied; on day 20,3-6 pups from each of 3 litters were studied; and on day 27, 3-6 pups from each of 3 litters were studied.

There was no day-night difference in SCN metabolic activity at day 16; the SCN were clearly visible on the autoradiographs and displayed greater metabolic activity than the surrounding hypothalamus during both subjective day and night (Figs. 6, 7). At $20 \mathrm{~d}$ of age, there was a clear day-night difference in SCN metabolic activity $(p<0.05$; Mann-Whitney test). High metabolic activity was observed in the SCN during subjective day, whereas at night, $\mathrm{SCN}$ metabolic activity appeared to be slightly greater than that of the adjacent hypothalamus. A clear daynight difference in metabolic activity was also observed in the SCN of 27-d-old pups $(p<0.01$; Mann-Whitney test). The nuclei were visible during the day, and, in contrast to days 16 


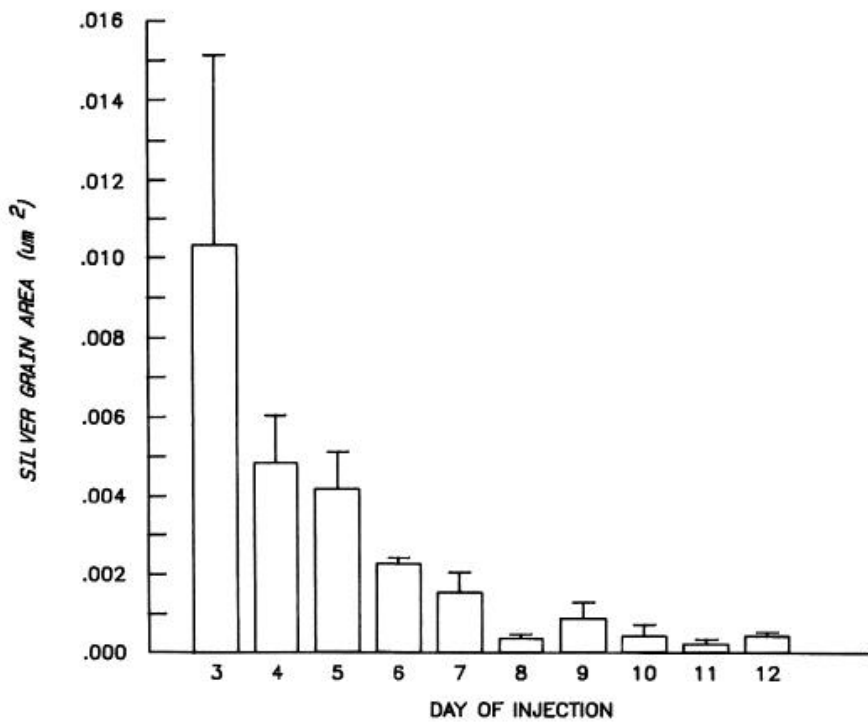

Figure 5. Quantitative analysis of the time course of $\mathrm{SCN}$ neurogenesis as determined by ${ }^{3} \mathrm{H}$-thymidine autoradiography. The height of the vertical bars is the mean area \pm SEM of silver grains overlying the left SCN in animals injected at different postnatal ages. Three to six animals were injected at each age.

and 20, the autoradiographic images of the SCN at day 27 were not visible at night. At 20 and $27 \mathrm{~d}$ of age, when a circadian oscillation of SCN metabolic activity was observed, the phase of metabolic activity appeared to be synchronous with both the estimated circadian time of the dam and the previous lightdark cycle.

\section{Discussion}

Our findings indicate that the gray short-tailed opossum has a functioning circadian timing system and that the daily lightdark cycle is an important entraining stimulus for opossum rhythms. Adult animals manifest robust daily rhythms in locomotor activity. Importantly, the locomotor activity rhythm persists in the absence of environmental cues (constant darkness), indicating that it is an endogenously generated circadian rhythm. Light-dark entrainment was shown when the daily lightdark cycle was reversed; the locomotor activity rhythm assumed a constant phase relationship with the new lighting cycle.

Circadian rhythms in locomotor activity have also been observed in other marsupials. The stripe-faced dunnart manifests clear day-night patterns of activity, and the rhythm responds to the entraining effects of light in a manner similar to that described for nocturnal rodents (Cassone, 1987). A circadian pattern in locomotor activity has also been observed in a predatory marsupial, the kowari (O'Reilly et al., 1986). The similarity of circadian characteristics of the locomotor activity rhythm between marsupials and rodents suggests that the underlying circadian system generating and regulating circadian rhythms is similar among these diverse mammalian species.

In eutherian species, lesion, metabolic, electrophysiologic, and pharmacologic studies indicate that the SCN are the site of a master circadian pacemaker in the mammalian brain (for review, see Takahashi and Zatz, 1982; Moore and Card, 1985). We provide 3 lines of evidence suggesting that the SCN are also a likely location for a circadian pacemaker in marsupials. First, our data show that the gray short-tailed opossum has a cluster of neurons in the anterior hypothalamus homologous to the $\mathrm{SCN}$ of eutherian animals. In fact, the SCN appear to be anatomically similar among many different orders of mammals,

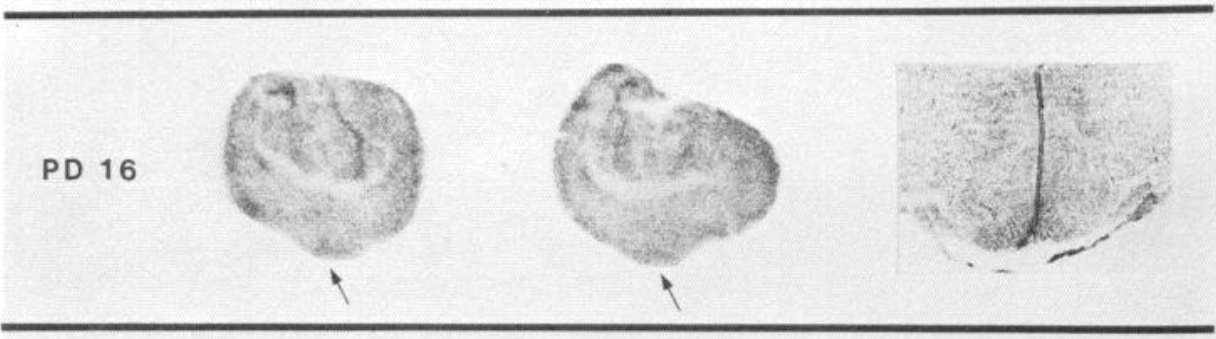

Figure 6. Autoradiographs of coronal brain sections from pups injected with dG during subjective day (left) and subjective night (middle) on postnatal days 16,20 , and 27 . The metabolically active SCN appear as dark spots during subjective day on days 16,20 , and 27 , and during subjective night on day 16 (arrows). Sections used to generate the autoradiographs in the middle column were stained with cresyl violet and are depicted on the right. Scale bars, $1 \mathrm{~mm}$.
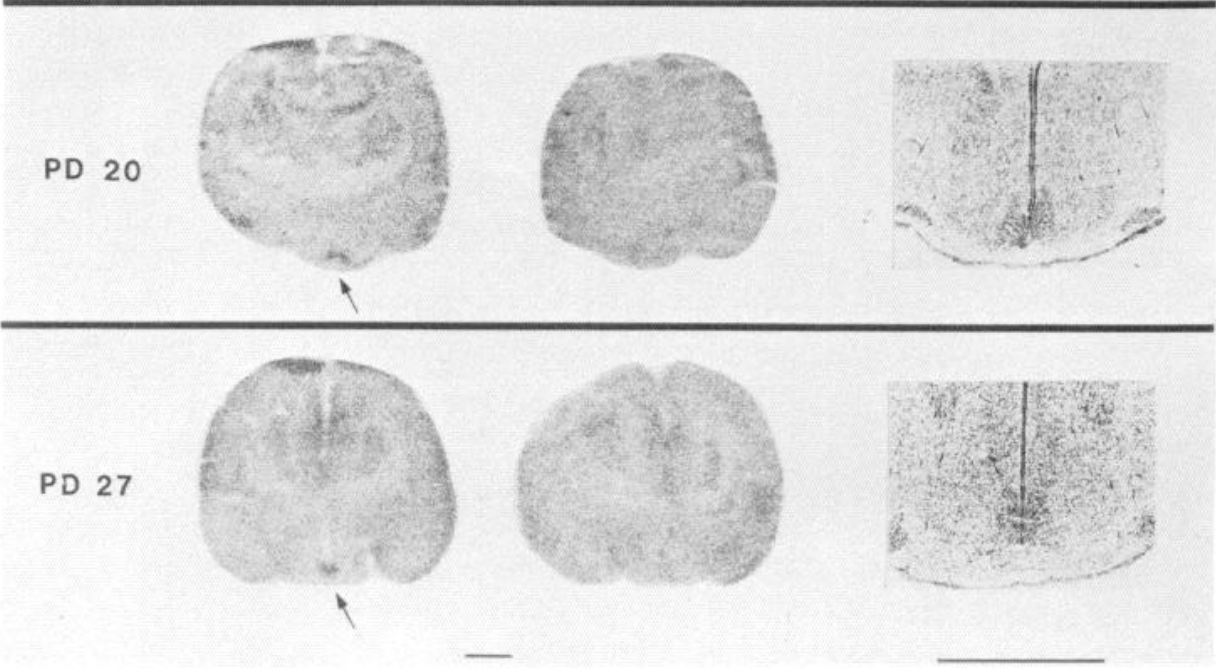
and the SCN of several marsupial species-in addition to the gray short-tailed opossum - have recently been described (V. M. Cassone, personal communication).

A second line of evidence supporting a circadian pacemaker function of the marsupial SCN is the existence of a direct retinohypothalamic tract from the retina to these nuclei. This tract is both necessary and sufficient for light-dark entrainment of circadian rhythms in eutherian mammals (Moore and Card, 1985). A retinohypothalamic tract has been described in several marsupial species (Moore, 1973; Pearson et al., 1976; Royce et al., 1976; Mendez-Otero et al., 1985), including the gray shorttailed opossum (V. M. Cassone, personal communication; S. A. Rivkees and S. M. Reppert, unpublished observations).

The most compelling evidence implicating the marsupial SCN as a circadian pacemaker is provided by our $\mathrm{dG}$ results. We demonstrate that the SCN of the adult gray short-tailed opossum exhibit a prominent daily rhythm in metabolic activity. Furthermore, the daily pattern of the metabolic activity rhythm in the opossum SCN is strikingly similar to that observed in rats, where the time course of the metabolic activity rhythm has been most extensively studied (Schwartz et al., 1980); in both species, metabolic activity in the SCN is high during subjective day and low during subjective night. The correspondence of the rhythms of SCN metabolic activity between the opossum and several eutherian species (Schwartz et al., 1983) suggests that the biochemical basis of SCN functional activity (which remains to be elucidated) is similar across mammalian phylogeny.

The qualitative similarities between our results in the opossum (in which dG was administered intraperitoneally) and the results in rats (in which $\mathrm{dG}$ was administered intravenously) validate the intraperitoneal route of administration as a way of monitoring circadian oscillations of SCN metabolic activity in the opossum. It is worth noting that the intraperitoneal and intravenous routes of $\mathrm{dG}$ delivery have been shown to be highly comparable for monitoring local cerebral glucose utilization in rats (Meibach et al., 1980).

A primary focus of this paper is the anatomic and functional development of the opossum SCN. Reflecting the profound degree of developmental immaturity of the newborn marsupial, the brain of the gray short-tailed opossum is not well differentiated and resembles a neural tube at birth. The hypothalamus is not apparent by light microscopy until postnatal day 3 (C. D. Jacobson, unpublished observations). Formation of SCN neurons occurs over the first $8 \mathrm{~d}$ of postnatal life (until postnatal day 7). It is not until nearly 1 week later (postnatal day 16) that the SCN are recognizable as distinct nuclei by light microscopy. This delay between final division and formation of recognizable nuclei is probably due to migration of SCN neurons from the site of formation (along the third ventricle) to their final location. The time frame between the termination of neurogenesis and the morphological identification of the SCN is similar to that described for other hypothalamic nuclei in the opossum (Larsen and Jacobson, 1985).

An interesting developmental pattern of circadian rhythmicity in SCN metabolic activity was observed in the opossum. On day 16 , when the $\mathrm{SCN}$ are first identifiable, the nuclei show increased metabolic activity during both the day and night. With increasing age, metabolic activity in the $\mathrm{SCN}$ appears to decrease at night, and, on day 20 , a significant day-night rhythm is first observed. One week later (day 27), nighttime metabolic activity in the SCN is no longer distinguishable from that of the adjacent hypothalamus. This suggests that the development of the cir-

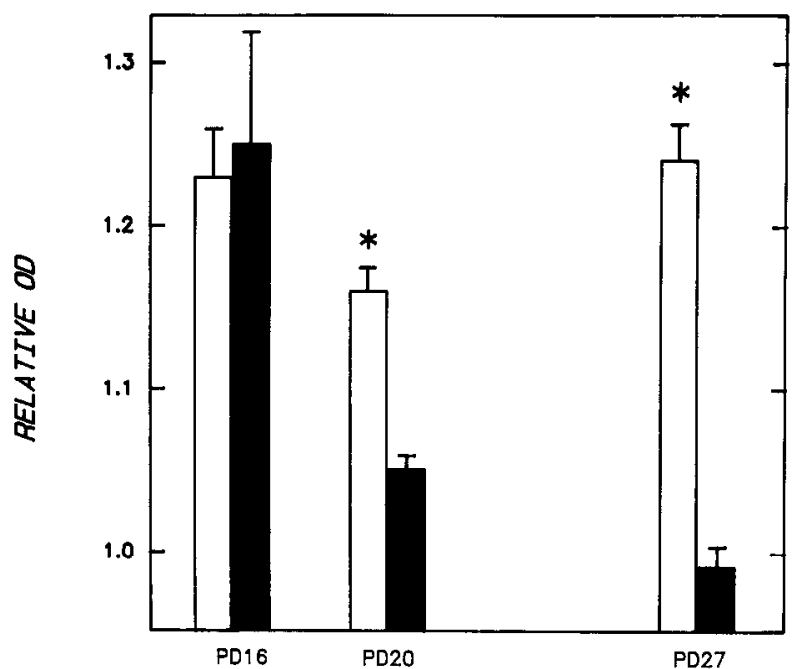

Figure 7. Relative optical densities of pup SCN (OD of SCN/OD of adjacent hypothalamus) during subjective day (open bars) and subjective night (solid bars) following dG injection on postnatal days (PD) 16, 20, and 27. The height of each bar is the mean relative OD \pm SEM of SCN for 11 or 12 pups obtained from $2-3$ litters at each age. A significant day-night difference is present on day 20 and day $27\left(^{*}, p<0.05\right.$; MannWhitney test).

cadian rhythm in SCN metabolic activity is attributable to a decrease in nighttime SCN metabolic activity. Alternatively, it is possible that an increase in the nighttime metabolic activity of the surrounding hypothalamus may contribute to the decline in the relative metabolic activity of the SCN at night.

In marked contrast to the opossum, in which early SCN development occurs during postnatal life, rats undergo these same developmental events in utero. The rat SCN undergo neurogenesis over days 12-16 of gestation (day of sperm positivity = day 0; Ifft, 1972; Altman and Bayer, 1978), and the nuclei are anatomically distinct on day 18 of gestation (Altman and Bayer, 1978). The rat SCN first exhibit a day-night rhythm in metabolic activity a day later (day 19 of gestation; $3 \mathrm{~d}$ before birth); a daynight difference in SCN metabolic activity is already present when the nuclei are first discernible from the surrounding tissue by their higher dG uptake (Reppert and Schwartz, 1983, 1984). Prenatal oscillations of SCN glucose utilization have also been observed in other eutherian species (Reppert, 1985; Weaver and Reppert, 1987).

Our data suggest that from its inception, the rhythm in SCN metabolic activity in the developing opossum is in phase with the "circadian time" of the dam and the prevailing LD cyclc. On the basis of studies in rodents, it is known that the dam entrains the developing SCN to the prevailing light-dark conditions in utero, and continues to influence the timing of the pup's circadian clock during the postnatal period (Reppert, 1985). Thus, maternal entrainment coordinates the phase of the developing biological clock to the environment until the time that the pups are directly responsive to external lighting through their own eyes.

Our study did not allow us to determine whether the coordination of pup circadian phase is mediated indirectly by the dam or directly by the environmental light-dark cycle. However, data from the tammar wallaby demonstrate that the retinohypothalamic tract forms late in the postnatal period, after the formation of most other visual pathways (Wye-Dvorak, 1984). It is thus tempting to speculate that entrainment is ma- 
ternally mediated in the marsupial during the early postnatal period, when the pup is continuously attached to the nipple of the dam and potentially unresponsive to light. Investigation of the relationships between these different entrainment mechanisms during opossum development should be enlightening.

\section{References}

Altman, J., and S. A. Bayer (1978) Development of the diencephalon in the rat. I. An autoradiographic study of the time of origin and settling patterns of neuron of the hypothalamus. J. Comp. Neurol. 182: 945-971.

Cassone, V. M. (1987) Circadian organization and photoreception in an Australian dasyurid marsupial (Smithopsis macroura). J. Biol. Rhythms 2: 261-268.

Cothran, E. G., M. J. Aivaliotis, and J. L. Vandeberg (1985) The effects of diet on growth and reproduction in gray short-tailcd opossums (Monodelphis domestica). J. Exp. Zool. 236: 103-1 14.

deGroot, J. (1959) The rat forebrain in stereotaxic coordinates. Verh. K. Akad. Geneeskd. Belg. 52: 1-40.

Fadem, B. H., G. L. Tripin, J. L. Vandeberg, and V. Hayssen (1982) Care and breeding of the gray, short-tailed opossum (Mondelphis domestica). Lab. Anim. Sci. 32: 405-409.

Hartman, C. G. (1952) Possums, pp. 71-81, U. Texas Press, Austin, TX.

Ifft, J. D. (1972) An autoradiographic study of the time of final division of neurons in the rat hypothalamic nuclei. J. Comp. Neurol. 144: 193-204.

Jacobson, C. D. (1984) Fetal mechanisms involved in the morphological sexual differentiation of the brain. In Fetal Neuroendocrinology, F. Ellcndorff, P. D. Gluckman, and N. Parvizi, eds., pp. 137148, Perinatology, New York.

Jacobson, C. D., and R. A. Gorski (1981) Neurogenesis of the sexually dimorphic nucleus of the preoptic area in the rat. J. Comp. Neurol. 196: 519-529.

Jacobson, C. D., F. C. Davis, and R. A. Gorski (1985) Formation of the sexually dimorphic nucleus of the preoptic area: Neuronal growth, migration and changes in cell number. Dev. Brain Res. 21: 1-18.

Larsen, R., and C. D. Jacobson (1985) Postnatal neurogenesis of the medial preoptic area in the gray short-tailed opossum (Monodelphis domestica). Anat. Rec. 214:71A.

Meibach, R. C., S. D. Glick, D. A. Ross, R. D. Cox and L. Mayani (1980) Intraperitoneal administration and other modifications of the 2-deoxy- $d$-glucose technique. Brain Res. 195: 167-176.

Mendez-Otero, R., L. $\Lambda$. Cavalcante, C. E. Rocha-Miranda, R. F. Bernardes, and P. C. R. Barradas (1985) Growth and restriction of ipsilateral retinocollicular projection in the opossum. Dev. Brain Res. 18: $199-210$.

Moore, R. Y. (1973) Retinohypothalamic projections in mammals: A comparative study. Brain Res. 49: 403-409.

Moore, R. Y., and J. P. Card (1985) Visual pathways and the entrainment of circadian rhythms. Ann. NY Acad. Sci. 453: 123-133.
Moore-Ede, M. C., F. M. Sulzman, and C. A. Fuller (1982) The Clocks That Time Us, pp. 31-113, Harvard U. P., Cambridge, MA.

O'Reilly, H. M., S. M. Armstrong, and G. J. Coleman (1986) Restrictcd feeding and circadian activity rhythms of a predatory marsupial, Dasyuroides byrnei. Physiol. Behav. 38: 471-476.

Pearson, L. J., K. J. Sanderson, and R. T. Wells (1976) Retinal projections in the ringtailed possum Pseudocheirus peregrinus. J. Comp. Neurol. 170: 227-240.

Pittendrigh, C. S., and S. J. Daan (1976) A functional analysis of circadian pacemakers in nocturnal rodents. I. The stability and lability of spontaneous frequency. J. Comp. Physiol. 106: 223-252.

Reppert, S. M. (1985) Maternal entrainment of the developing circadian system. Ann. NY Acad. Sci. 453: 162-169.

Reppert, S. M., and W. J. Schwartz (1983) Maternal coordination of the fetal biological clock in utero. Science 220:969-971.

Reppert, S. M., and W. J. Schwartz (1984) The suprachiasmatic nuclei of the fetal rat: Characterization of a functional circadian clock using ${ }^{14} \mathrm{C}$-labeled deoxyglucose. J. Neurosci. 4: 1677-1682.

Reppert, S. M., M. J. Duncan, and D. R. Weaver (1987) Maternal influences on the developing circadian system. In Perinatal Development: A Psychobiological Perspective, N. A. Krasnegor, E. Blass, M. Hofer, and W. Smotherman, pp. 343-356, Academic, Orlando, FL.

Royce, G. C., J. P. Ward, and J. K. Harting (1976) Retinalfugal pathways in two marsupials. J. Comp. Neurol. 170: 391-414.

Schwartz, W. J., and H. Gainer (1977) Suprachiasmatic nucleus: Use of ${ }^{14} \mathrm{C}$-labeled dexoyglucose uptake as a functional marker. Science 197: 1089-1091.

Schwartz, W. J., L. C. Davidson, and C. B. Smith (1980) In vivo metabolic activity of a putative circadian oscillator, the rat suprachiasmatic nucleus. J. Comp. Neurol. 198: 156-167.

Schwartz, W. J., S. M. Reppcrt, S. M. Eagan, and M. C. Moore-Ede (1983) In vivo metabolic activity of the suprachiasmatic nucleus: A comparative study. Brain Res. 273: 184-187.

Sharp, F. R., T. S. Kilduff, S. Bzorgchami, C.. H. Heller, and A. F. Ryan (1983) The relationship of local cerebral glucose utilization to optical density ratios. Brain Res. 263: 97-104.

Sokoloff, L. M., C. Reivich, M. H. Kennedy, M. H. DesRosiers, M. H. Patlak, K. D. Pettigrew, O. Sakurada, and M. Shinohar (1977) The $\left[{ }^{14} \mathrm{C}\right]$ deoxyglucose method for the measurement of local cerebral glucose utilization: Theory, procedure, and normal values in conscious and anesthetized albino rats. J. Neurochem. 28: 897-916.

Takahashi, J. S., and M. Zatz (1982) Regulation of circadian rhythmicity. Science 217: 1104-1111.

Van den Pol, A. N. (1980) The hypothalamic suprachiasmatic nucleus of rat: Intrinsic anatomy. J. Comp. Neurol. 191: 661-702.

Weaver, D. R., and S. M. Reppert (1987) Maternal-petal communication of circadian phase in a precocious rodent, the spiny mouse. Am. J. Physiol. 253: 401-409.

Wye-Dvorak, J. (1984) Postnatal development of primary visual projections in the tammar wallaby (Macropus eugenii). J. Comp. Neurol. 228: 491-508. 